Enferm Bras 2019;18(1):95-104

http://dx.doi.org/10.33233/eb.v18i1.2704

\title{
ARTIGO ORIGINAL \\ Acessibilidade para pessoas com deficiência na atenção básica de saúde em Três Rios, Centro Sul Fluminense
}

Renata Pedreira da Cruz, M.Sc. ${ }^{*}$, Rafael André da Silva, M.Sc. ${ }^{*}$, Juarez de Souza Pereira **, Vanessa Vianna Cruz ${ }^{* * *}$, Luciana Krauss Rezende ${ }^{* *}$, Ricardo da Silva Monteiro***, Wiliam César Alves Machado*****

*Enfermeiro, UNIRIO, ${ }^{* *} F i s i o t e r a p e u t a$, Professor na Faculdade Vértice de Três Rios (UNIVÉRTIX), ${ }^{* * * E n f e r m e i r a, ~ M e s t r a n d a ~ n o ~ P r o g r a m a ~ d e ~ P o ́ s-G r a d u a c ̧ a ̃ o ~ e m ~ E n f e r m a g e m ~}$ PPGENF - UNIRIO, ${ }^{* * * * A}$ Arquiteto, Professor na Faculdade Vértice de Três Rios (UNIVÉRTIX), *****Enfermeiro, Professor na Universidade Federal do Estado do Rio de Janeiro (UNIRIO), Faculdade Vértice de Três Rios (UNIVÉRTIX)

Recebido em 20 de dezembro de 2018; aceito em 13 de fevereiro de 2019.

Endereço para correspondência: Wiliam César Alves Machado, wilmachado@uol.com.br; Renata Pedreira da Cruz: renatapcrj@gmail.com; Rafael André da Silva: rafael1853@hotmail.com; Juarez de Souza Pereira: juarez.fisio@gmail.com; Vanessa Vianna Cruz: vanessavianna2005@yahoo.com.br; Luciana Krauss Rezende: lukrare@uol.com.br; Ricardo da Silva Monteiro: ricardo@rodriguesmonteiro.com.br

\section{Resumo}

Objetivo: Analisar as condições de acessibilidade para as pessoas com deficiência nas unidades de atenção básica de saúde do município de Três Rios, Rio de Janeiro, Brasil. Métodos: Estudo exploratório, com abordagem quantitativa, realizado com estudantes de PósGraduação, no primeiro semestre de 2016, utilizando um questionário autoaplicável em 29 estabelecimentos de saúde: 28 unidades básicas de saúde e um complexo regulador. Os resultados foram analisados a luz da estatística descritiva, e discutidos com base no preconizado pelos parâmetros antropométricos oficiais vigentes, no Brasil. Resultados: 51,7\% da amostra (15 unidades) não apresentavam condições mínimas de acessibilidade para pessoas com deficiência, enquanto outras 14 dispunham de algumas condições básicas. Em 6 delas os usuários alcançavam a recepção, em apenas 2 existia vão de portas para acesso de cadeirantes; 4 com acesso à sala de atendimentos ao público, aos consultórios, aos banheiros masculino e feminino; apenas 9 delas ofereciam condições transitáveis nos entornos. Acessibilidade para surdos e cegos, também inexpressíveis. Conclusão: Conclui-se que as unidades básicas de saúde estudadas não estão preparadas para acolher com dignidade cidadãos e cidadãs com deficiência ou mobilidade reduzida, o que demonstra flagrante descumprimento da legislação vigente e descaso dos gestores públicos para com as necessidades de cuidados e saúde para essas pessoas.

Palavras-chave: acesso aos serviços de saúde, atenção básica de saúde, estruturas de acesso, pessoas com deficiência.

\footnotetext{
Abstract

Accessibility to disabled persons in primary health care units in Três Rios, Rio de Janeiro, Brazil

Objective: To analyze the accessibility conditions for disabled person in the basic health care units of the municipality of Três Rios, Rio de Janeiro, Brazil. Methods: Exploratory study with quantitative approach, carried out with master graduate students, in the first half of 2016, using a self-administered questionnaire in 29 health facilities: 28 basic health units and a regulatory complex. The results were analyzed in the light of the descriptive statistics, and discussed based on the one recommended by the official anthropometric parameters in force in Brazil. Results: $51.7 \%$ of the sample (15 units) did not present minimum accessibility conditions for disabled person, while another 14 had some basic conditions. In 6 of them users reached the reception, in only 2 there was a doorway for wheelchair users; 4 with access to the reception room to the public, to the offices, to the men's and women's bathrooms; only 9 of them offered
} 
passable conditions in the surroundings. Accessibility for deaf and blind, were also inexpressible. Conclusion: The basic health units studied are not prepared to welcome for disabled citizens or with reduced mobility, which demonstrates flagrant noncompliance with current legislation and public managers' disregard for the health and care needs of these people. Key-words: health services accessibility, primary health care, architectural accessibility, disabled person.

\section{Resumen}

Accesibilidad para personas con discapacidad en la atención básica de salud en Três Rios, Río de Janeiro, Brasil

Objetivo: Analizar las condiciones de accesibilidad para las personas con discapacidad en las unidades de atención básica de salud del municipio de Três Rios, Río de Janeiro, Brasil. Métodos: Estudio exploratorio, con abordaje cuantitativo, realizado con estudiantes de posgraduación en salud, en el primer semestre de 2016, utilizando un cuestionario autoaplicable en 29 establecimientos de salud: 28 unidades básicas de salud y un complejo regulador. Los resultados fueron analizados a la luz de la estadística descriptiva, y discutidos con base en el preconizado por los parámetros antropométricos oficiales vigentes, en Brasil. Resultados: $51,7 \%$ de la muestra (15 unidades) no presentaban condiciones mínimas de accesibilidad para las personas con discapacidad, mientras que otras 14 tenían algunas condiciones básicas. En 6 de ellas los usuarios alcanzaban la recepción, en apenas 2 existía van de puertas para acceso de usuarios de sillas de ruedas; 4 con acceso a la sala de atención al público, a los consultorios, a los baños masculino y femenino; sólo 9 de ellas ofrecían condiciones transitable en los entornos. Accesibilidad para sordos y ciegos, también inexpresables. Conclusión: Se concluye que las unidades básicas de salud estudiadas no están preparadas para acoger con dignidad las personas con discapacidad o movilidad reducida, lo que demuestra flagrante incumplimiento de la legislación vigente y descuido de los gestores públicos para con las necesidades de atención y salud para esas personas.

Palabras-clave: accesibilidad a los servicios de salud, atención primaria de salud, estructuras de acceso, personas con discapacidad.

\section{Introdução}

A população de pessoas com deficiência é composta de um grupo heterogêneo, pois reúne indivíduos com deficiência física, auditiva, visual e intelectual e, desse modo, há a necessidade de garantir a esse público acesso as ações e serviços de saúde considerando suas características e diferentes necessidades [1].

Segundo o Censo do IBGE de 2010, entre mais de 190 milhões de habitantes, 23,9\% da população do Brasil apresenta um tipo de deficiência, com maior ocorrência da deficiência visual, afetando $18,6 \%$ da população do país, seguida da deficiência motora (7\%), deficiência auditiva $(5 \%)$ e da deficiência mental ou intelectual $(1,40 \%)$ [2].

A oferta de ambientes com acessibilidade é uma exigência constitucional, tem como objetivo permitir ganhos de autonomia e de mobilidade a uma porção maior de pessoas, incluindo aquelas que tenham dificuldades de locomoção [3]. No caso deste estudo, para que pessoas com deficiência ou mobilidade reduzida, os usuários dos serviços disponíveis nas unidades básicas de saúde possam deles usufruir com mais segurança, confiança e comodidade.

A associação do aspecto sócio-organizacional com o geográfico da acessibilidade gera barreiras físicas, arquitetônicas e de comunicação que impedem que pessoas com deficiência exerçam seus direitos de acesso ao tratamento em igualdade de condições, quando comparado às pessoas sem deficiência [4]. O enfrentamento cotidiano dessas barreiras põe em risco a saúde e o processo de reabilitação das primeiras, afetando negativamente a necessidade de cuidado de longo prazo dessas pessoas [3].

A Associação Brasileira de Normas Técnicas (ABNT) em 2015 revisou e atualizou a Norma Brasileira (NBR) 9050/2015 [5], que regulamenta e define os critérios e parâmetros sobre acessibilidade a edificações, mobiliário, espaços e equipamentos urbanos. Define acessibilidade como possibilidade e condição de alcance, percepção e entendimento para a utilização com segurança e autonomia em edificações, espaços, mobiliários e a equipamentos urbanos. Estabelece as normas gerais de construções, adaptações e adequações para que todas as pessoas tenham acesso facilitado em qualquer local público no país. Importante para este estudo são seus parâmetros, que incluem rota externa e interna acessível, sinalização 
visual e tátil, mobiliários, banheiros e estacionamento dentro dos padrões de acessibilidade, entre outros.

Da mesma forma relevante para este estudo, os elementos do Decreto ํo 6.949 [6], de 25 de agosto de 2009, que promulga a Convenção Internacional sobre os Direitos das Pessoas com Deficiência e seu Protocolo Facultativo, e norteia questões referentes aos direitos de pessoas com deficiência, estabelece normas gerais e critérios básicos para a promoção da acessibilidade destas pessoas, garantindo que elas tenham acesso igualitário a todos os direitos concedidos por lei.

A legislação brasileira garante a todos os cidadãos, incluindo as pessoas com deficiências, o direito ao acesso à educação, à saúde, ao lazer e ao trabalho. Ademais, o conceito de cidadania envolve o termo acessibilidade, no qual os indivíduos têm direitos assegurados por lei que devem ser respeitados, entretanto muitos destes direitos esbarram em barreiras arquitetônicas, de comunicação, além de barreiras sociais [7].

Com a organização dos serviços de saúde do Sistema Único de Saúde em redes de atenção, as Unidades Básicas de Saúde (UBS) são a porta de entrada preferencial do sistema para todos os usuários, inclusive os deficientes, terem acesso às redes de atenção. As condições de acessibilidade nas UBS, como em todos os espaços arquitetônicos e urbanísticos, citados em lei, devem envolver condições de acesso para pessoas com deficiência física, visual e auditiva, de modo a atender igualitariamente todos os cidadãos [8].

Diante do exposto e do fato da escassez de estudos sobre acessibilidade principalmente em UBS, este relato de experiência tem o objetivo de analisar as condições de acessibilidade para as pessoas com deficiência nas unidades de atenção básica de saúde do Município de Três Rios, região Centro Sul do Estado do Rio de Janeiro.

\section{Material e métodos}

Trata-se de estudo exploratório, com abordagem quantitativa, realizado no primeiro semestre de 2016. O cenário deste estudo compreendeu as Unidades Básicas de Saúde (UBS) e o Complexo Regulador do Município de Três Rios, Rio de Janeiro, Brasil. A amostra foi composta por 28 UBS que desenvolvem a Estratégia de Saúde da Família para atender munícipios de Três Rios e o seu Complexo Regulador, somando 29 unidades de saúde.

O Complexo Regulador foi escolhido por ser o espaço onde as pessoas buscam informações gerais acerca do Sistema de Saúde no Município, devendo o mesmo ser tão acessível quanto as UBS.

Os dados foram coletados no primeiro semestre de 2016, em visitas às unidades, devidamente acompanhadas de seus gestores. Com ajuda desses, foi preenchido instrumento, contendo questões referentes à identificação da unidade de saúde e aspectos referentes à acessibilidade para usuário com mobilidade reduzida ou pessoa com deficiência, baseado nos parâmetros da NBR 9050/2015 [5].

As questões avaliaram o acesso à recepção nas unidades, o acesso às instalações internas de atendimento e consultórios, a acessibilidade aos banheiros femininos e masculinos, a largura dos vãos das portas, rotas acessíveis interna e externa, sinalizações, entre outras questões que podem otimizar ou limitar acesso dos usuários com deficiência ou mobilidade reduzida. Esses dados foram transcritos para o banco de dados como adequados ou não aos parâmetros da NBR 9050/2015 [5].

Ainda no questionário buscou-se identificar se algum servidor da unidade domina a Língua de Sinais Brasileira (Libras), se existe sinalização tátil no piso/parede/quadros em Braile, para orientação espacial de cegos.

Dados foram submetidos ao software SPSS (Statistical Package for the Social Sciences version 21) para análise estatística descritiva simples. Com os resultados obtidos foi possível discutir com parâmetros antropométricos da ABNT, discutidos a partir dos resultados de estudos que abordam a temática, disponíveis nas bases de dados da área de conhecimento.

A experiência constitui contribuição para ampliar a aproximação e melhor compreensão da temática - acessibilidade de pessoas com algum tipo de deficiência - aos serviços de saúde da Atenção Básica, para estudantes de Graduação e Pós-Graduação, gestores públicos, docentes e pesquisadores que se dedicam ao estudo de fenômenos relacionados.

Da mesma forma, procura elucidar explicações para que tamanhas disparidades excludentes ainda persistam nos diversos ambientes de cuidado à saúde, a despeito da legislação vigente no Brasil. 
Consoante com a Resolução 466/20129 do CNS, foi extraído de estudo submetido ao Comitê de Ética da Universidade Federal do Estado do Rio de Janeiro, via Plataforma Brasil, e aprovado com o parecer de no 1.241.940 em 23/09/2015, CAAE 45409015.4.0000.5285.

\section{Resultados}

Foram analisados 28 UBS e o Complexo Regulador do município de Três Rios - RJ, totalizando 29 unidades de saúde, onde se priorizou avaliar a acessibilidade das unidades em relação aos parâmetros sobre acessibilidade a edificações, mobiliário, espaços e equipamentos urbanos que foram definidos e regulamentados através da NBR 9050/2015 [5]. Os aspectos destacados foram o acesso à recepção, a sala de atendimento, aos consultórios, aos banheiros femininos e masculinos, medidas das larguras das portas, sobre a possibilidade de comunicação através da Libras, das condições das rotas interna e externa e sobre a existência de placas sinalizadoras em Braile.

As barreiras arquitetônicas ou de comunicação comprometem negativamente o direito de acesso das pessoas com mobilidade reduzida ou algum tipo de deficiência, neste estudo, o direito de acesso aos serviços básicos de saúde, infringindo princípios constitucionais do Brasil, como pode ser observado na tabela I.

Tabela I - Condições fundamentais de acessibilidade por tipo de deficiência. Unidades Básicas de Saúde. Três Rios/RJ, 2016.

\begin{tabular}{|c|c|c|c|}
\hline Condições de acesso & $\begin{array}{l}\text { Deficiência física ou } \\
\text { mobilidade reduzida }\end{array}$ & $\begin{array}{l}\text { Deficiência } \\
\text { visual }\end{array}$ & $\begin{array}{l}\text { Deficiência } \\
\text { auditiva }\end{array}$ \\
\hline Acesso à recepção & $x$ & $x$ & $x$ \\
\hline Acesso à sala de atendimento & $x$ & $x$ & $\mathrm{X}$ \\
\hline Acesso aos consultórios & $x$ & $x$ & $x$ \\
\hline Acesso ao banheiro feminino & $x$ & $x$ & $x$ \\
\hline Acesso ao banheiro masculino & $x$ & $x$ & $x$ \\
\hline Largura adequadas das portas & $x$ & & \\
\hline Condição do entorno & $X$ & $x$ & $x$ \\
\hline Sinalizadores/ placa em braile & & $x$ & \\
\hline Comunicação em Libras & & & $x$ \\
\hline Banheiros com barras & $x$ & & \\
\hline $\begin{array}{l}\text { Bacias sanitárias de acordo com as } \\
\text { normas }\end{array}$ & $x$ & & \\
\hline $\begin{array}{l}\text { Balcão rebaixado para atender } \\
\text { cadeirante }\end{array}$ & $x$ & & \\
\hline $\begin{array}{l}\text { Disponibilidade de servidor que } \\
\text { domina Libras ou presença de } \\
\text { intérprete }\end{array}$ & & & $x$ \\
\hline
\end{tabular}

A seguir a Tabela II apresenta a frequência encontrada sobre as condições de acessibilidade nas 29 unidades de saúde analisadas e o respectivo percentual de acessibilidade. 
Tabela II - Condições de acessibilidade encontradas nas Unidades Básicas de Saúde do Município de Três Rios/RJ, 2016.

\begin{tabular}{llll}
\hline Categorias & & Frequência absoluta & Frequência relativa \\
\hline $\mathrm{I}$ & Acesso à recepção & 6 & $15 \%$ \\
\hline $\mathrm{II}$ & $\begin{array}{l}\text { Acesso à sala de } \\
\text { atendimento }\end{array}$ & 4 & $10 \%$ \\
\hline $\mathrm{III}$ & Acesso aos consultórios & 4 & $10 \%$ \\
\hline $\mathrm{IV}$ & $\begin{array}{l}\text { Acesso ao banheiro } \\
\text { feminino }\end{array}$ & 4 & $10 \%$ \\
\hline $\mathrm{V}$ & $\begin{array}{l}\text { Acesso ao banheiro } \\
\text { masculino }\end{array}$ & 4 & $10 \%$ \\
\hline $\mathrm{VI}$ & $\begin{array}{l}\text { Largura adequada das } \\
\text { portas }\end{array}$ & 2 & $5 \%$ \\
\hline $\mathrm{VII}$ & Condições do entorno & 9 & $22,5 \%$ \\
\hline $\mathrm{VIII}$ & Placas em Braile & 2 & $5 \%$ \\
\hline $\mathrm{IX}$ & Comunicação em Libras & 5 & $12.5 \%$ \\
\hline & total & $\mathbf{4 0}$ & $\mathbf{1 0 0 \%}$ \\
\hline
\end{tabular}

A tabela III apresenta a relação entre o número de unidades com a frequência de critérios e o tipo de condição de acessibilidade presente nos estabelecimentos avaliados.

Tabela III - Unidades de Saúde e itens de acessibilidade que dispõem. Três Rios/ RJ, 2016.

\begin{tabular}{|c|c|c|c|}
\hline $\begin{array}{l}\text { Unidades de } \\
\text { saúde }\end{array}$ & $\begin{array}{l}\text { Frequência de } \\
\text { critérios }\end{array}$ & $\begin{array}{l}\text { Condição de acessibilidade } \\
\text { disponível }\end{array}$ & \\
\hline 15 unidades & 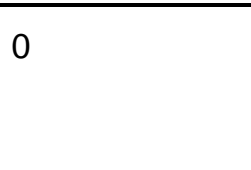 & $\begin{array}{l}\text { Não Dispõem de condição de } \\
\text { acessibilidade } \\
1 \text { unidade apresenta disponível a } \\
\text { condição I }\end{array}$ & $51 \%$ \\
\hline 9 unidades & 1 & $\begin{array}{l}4 \text { unidades apresentam disponível a } \\
\text { condição VII } \\
4 \text { unidades apresentam disponível a } \\
\text { condição IX }\end{array}$ & $31 \%$ \\
\hline 1 unidade & 2 & $\begin{array}{l}1 \text { unidade apresenta disponível as } \\
\text { condições I e VII } \\
\text { Duas unidades apresentam }\end{array}$ & $3.4 \%$ \\
\hline 2 unidades & 6 & $\begin{array}{l}\text { disponíveis as condições I, II, III, IV, } \\
\text { V e } \\
\text { VII }\end{array}$ & $6.9 \%$ \\
\hline 2 unidades & 8 & $\begin{array}{l}\text { Duas unidades apresentam } \\
\text { disponíveis as condições I, II, III, IV, } \\
\text { V, VI, VIII e IX }\end{array}$ & $6.9 \%$ \\
\hline
\end{tabular}

I (Acesso à recepção), II (Acesso a sala de atendimento), III (Acesso aos consultórios), IV (Acesso ao banheiro feminino), V (Acesso ao banheiro masculino), VI (Largura adequadas das portas), VII (Condição do entorno), VIII (Sinalizadores/ placa em Braile) e IX (Comunicação em Libras).

Observou-se que a maioria das unidades $(51,7 \%)$ não dispunha de nenhum item de acessibilidade exigido pela NBR 9050 de 2015 [5]. Das 29 unidades de saúde, nove apresentavam apenas um item de acesso disponível em suas unidades, quatro delas dispunham de um funcionário que dominava a Língua de Sinais Brasileira (Libras), quatro unidades apresentavam condições de entorno favoráveis de forma que os usuários, inclusive os com deficiência, podiam acessar com independência.

Uma unidade apresentava acessibilidade satisfatória na recepção e apenas duas unidades $(6,9 \%)$ cumpriam oito itens exigidos na norma vigente e outras duas $(6,9 \%)$ disponibilizavam em suas unidades seis itens importantes para facilitar o acesso das pessoas com deficiência ao serviço de Atenção Básica de Saúde no município.

Dos nove itens questionados, 25 unidades $(86,2 \%)$ apresentavam menos que $25 \%$ de cumprimento às exigências de condições de acessibilidade de acordo com as normas da NBR 
9050/2015 [5] e apenas quatro unidades apresentavam mais que 67\% adequação as condições de acessibilidade.

Discussão

Devido às peculiaridades da própria deficiência somadas as comorbidades, os indivíduos com deficiência necessitam utilizar os serviços de saúde e a acessibilidade a esses serviços e sua satisfação estão diretamente associadas aos obstáculos que podem inviabilizar o seu acesso [10].

Nenhuma das UBS desta pesquisa cumpre com todos os critérios e parâmetros sobre acessibilidade a edificações, mobiliário, espaços e equipamentos urbanos que foram determinados pela NBR 9050/2015 [5]. A maioria (51,7\%) das unidades não apresentou nenhuma das condições de acessibilidade em suas edificações e 48,3\% apresentou poucas condições de acesso para as pessoas com deficiência.

A dificuldade de acesso às UBS nos demonstra o conflito existente entre a legislação que assegura acessibilidade das pessoas com deficiência a Saúde e os pressupostos da NBR 9050/2015 [5], além de confrontar com as diretrizes de humanização, que priorizam o respeito e o atendimento humanizado, de forma acolhedora e resolutiva [11].

É importante destacar que baixas condições de acessibilidade também têm sido encontradas em outros níveis de atenção. Em uma pesquisa realizada em hospitais no município de João Pessoa, na Paraíba, foi constatado que nessas instituições de saúde havia a existência de barreiras arquitetônicas implicando no acesso aos serviços de saúde [12].

O fato é que a NBR 9050 [5] está em vigor desde 2015, assegurando que todos os espaços, edificações, equipamentos urbanos devem ser acessíveis de maneira que estes possam ser alcançados, acionados, utilizados e vivenciados por qualquer pessoa, inclusive aquelas com mobilidade reduzida. Essa Norma chama atenção para que o termo acessível implique tanto em acessibilidade física como de comunicação, o que envolve acessibilidade para os deficientes físicos ou pessoas com mobilidade reduzida e deficientes visuais e auditivos. Essa norma determina ainda que as condições de acesso e circulação interna nas unidades também precisam estar de acordo para garantir que as pessoas possam desenvolver suas atividades com normalidade e independência.

A falta de acessibilidade não é uma particularidade nas unidades de saúde, elas podem ser observadas na sociedade de uma forma geral, o que reflete numa grave omissão do Estado em garantir e cumprir com o que se encontra legalizado. Esse fato vai ao encontro a achados em um estudo realizado através de Oficinas Vivenciais do projeto "Vivenciando a Deficiência: (des) educando para incluir", realizada junto a 638 alunos matriculados na disciplina Psicologia da Educação, na Universidade Federal de Uberlândia (MG), evidenciando que mesmo após reformas estruturais, as instalações das IES não atendem aos requisitos para a acessibilidade das pessoas com deficiência exigidos, tanto aspectos urbanísticos, como arquitetônicos e de informação e comunicação (sinalização, sistemas de consulta e empréstimos, tecnologia de apoio para usuários portadores de deficiências, sistemas para acesso remoto etc.) precisam ser adaptados [13].

Considerando a equiparação de oportunidades, todos os seres humanos devem ter igualdade de oportunidades em participar de todas as atividades da vida e usufruir de bens e serviços, se faz necessário que as vias e espaços públicos sejam transformados em ambientes acessíveis para garantir este direito às pessoas com deficiência ou aquelas com mobilidade reduzida [14].

Um dos critérios de acessibilidade analisado no presente estudo foi avaliar as condições do entorno. Esse critério se aproxima do termo rota acessível presente na NBR 9050/2015 [5] que trata como trajeto contínuo, desobstruído e sinalizado, conectando ambientes externos (estacionamentos, calçadas rebaixadas, faixas de travessia de pedestres, rampas, etc.) ou internos (corredores, pisos, rampas, escadas, elevadores etc.).

As condições do entorno das unidades são importantes para garantir o pleno funcionamento do serviço, todas as entradas devem ser acessíveis bem como as rotas de interligação às principais funções do edifício. As áreas de circulação externa devem ser utilizadas de forma autônoma e seguras por todas as pessoas, inclusive as com deficiência.

No entanto, quando se observa o resultado a respeito das condições do entorno nas unidades de saúde pesquisadas, objeto deste estudo, apenas $32 \%$ delas é acessível considerando esse critério como condição indispensável para que as pessoas com deficiência possam ter acesso às UBS e consequentemente aos serviços que elas disponibilizam. Em 
contrapartida, foi realizado estudo, em 2011, com 100 famílias cadastradas no Sistema de Informação da Atenção Básica (SIAB), moradores da área de abrangência da Unidade de Saúde Américo Bertão, Jardim Eldorado, município de Cordeirópolis, SP, Brasil [15], com objetivo de identificar os fatores associados ao acesso a serviços básicos de saúde partindo do pressuposto de que fatores atrelados a diferentes dimensões do indivíduo e dos serviços de saúde se relacionam na sua construção. Os resultados mostram que os fatores associados ao acesso dos moradores ao serviço no qual estão adstritos são: a naturalidade; o uso da unidade de saúde na época; o local de residência; e a classificação do atendimento da unidade. Contudo, em se tratando da garantia de calçadas que garantissem o deslocamento seguro de deficientes visuais, cadeirantes, idosos e outras pessoas com mobilidade reduzida, no presente estudo, dados revelaram que foram consideradas como inexistentes em $66,7 \%$ das UBS.

Os resultados obtidos sobre os critérios de acessibilidade no ambiente interno deste estudo apresentaram que o acesso à sala de atendimento, aos consultórios e aos banheiros (femininos e masculinos) apenas $13,79 \%$ das unidades garantem acessibilidade nesses espaços e $20,69 \%$ tem recepção acessível. Dificuldades no acesso a serviços de saúde também foram observadas em outro estudo, no qual se observou que não havia rampas, inexistência de elevadores e de sinalização para as pessoas com deficiência. Além de lugares de espera insuficientes e salas com presença de obstáculos físicos impedindo o acesso dessas pessoas, e ainda ausência ou inadequação de sanitários destinados às pessoas com deficiência [10].

Apenas $6,9 \%$ das UBS atendem às exigências de larguras das portas. De acordo com a NBR 9050/2015 [5], as portas devem ter no mínimo vão livre de 0,80 m e altura de 2,10 m e devem permitir a aproximação de cadeira de rodas. As informações essenciais aos espaços internos e externos das edificações devem ser sinalizadas de forma visual, tátil, gestual e sonora [5].

O resultado obtido neste estudo também nos apontou que apenas $17,24 \%$ das UBS dispõem de profissionais capazes de se comunicarem em Libras, e apenas 6,9\% cumpre com a existência de placas de Braile. Um estudo realizado sobre deficiência como restrição de participação social e os desafios para avaliação a partir da Lei Brasileira de Inclusão [16], em diversos setores da sociedade, constatou em sua maioria ausência de informações em braile, sonoras ou visuais. Observa-se, assim, que não somente unidades de saúde descumprem as normas sobre acessibilidade e permitem que barreiras de comunicação impeçam que pessoas com deficiência visual ou auditiva possam desenvolver com autonomia e independência suas atividades [3].

A pesquisa demonstrou que instalações das UBS não estão adequadas para atender ao Decreto 6.949/2009 [6], bem como a NBR 9050/2015 [5]. Resultados semelhantes foram encontrados no estudo que verificou a acessibilidade nas UBS em um município do estado da Bahia, que identificou que aproximadamente dois terços dos prédios não eram adequados às necessidades dos usuários [17]. Esses autores afirmaram que muitas unidades eram instaladas em edificações improvisadas disponíveis nas comunidades e não específicas para atenderem as necessidades dos profissionais e as dos usuários [17]. Tal fato não difere do que foi encontrado no presente estudo.

$\mathrm{Na}$ realidade, parte dos imóveis são construções antigas disponíveis nas comunidades, alugadas pelo poder público municipal e adaptadas para instalar as unidades de saúde, o que pode explicar, em parte, as inadequações arquitetônicas encontradas em seu interior. Porém, o Decreto no 6.949/2009 [6] considera que o Poder Público buscará garantir dotação orçamentária para ampliar o número de acessos nas edificações de uso público a serem construídas, ampliadas ou reformadas, e que as edificações de uso público e de uso coletivo referidas, já existentes, têm, respectivamente, prazo de trinta e quarenta e oito meses, a contar da data de publicação do Decreto, para garantir a acessibilidade de que trata este Decreto. Deste modo, observamos que tais UBS já deveriam estar adaptadas e acessíveis para pessoas com deficiência.

Tal fato também é confirmado por estudo sobre acessibilidade de pessoas com deficiência ou restrição permanente de mobilidade ao SUS [18], revelando que maior parte das Unidades Básicas de Saúde está instalada em casas improvisadas, um tipo de construção que não atende às necessidades dos usuários; e outro estudo também aponta que os prédios das UBS não eram adequados para o acesso de idosos e pessoas com deficiência física [19].

O Ministério da Saúde tem buscado estratégias para mudar essa realidade através de portarias como a de № 339 de 2013, que redefine o componente ampliação do Programa de Requalificação de Unidades Básicas de Saúde. Esse programa tem o objetivo de fortalecer a 
atenção básica através da estruturação de suas unidades, mediante a transferência de recursos financeiros direto da esfera federal para os municípios. Visa também transformar as UBS em estabelecimentos acolhedores melhorando o acesso, facilitando a prática das equipes de saúde e buscando uma melhor qualidade do atendimento [20]. No entanto, ele não tem sido capaz de mudar a realidade encontrada pela dificuldade do município cumprir com algumas exigências. Pois, tanto para ampliar, reformar, assim como para construir com esses recursos os imóveis devem ser próprios dos municípios ou cedidos por outro ente federado.

Considerando, ainda, que as pessoas com deficiência devam fazer uso deste espaço em igualdade de condições aos demais usuários, é importante que mudanças ocorram na forma da sociedade se organizar para que se possa tratar a deficiência com mais justiça e igualdade [17].

Cabe destacar a inexistência de fiscalização efetiva que exija do poder público e do setor privado o cumprimento do Decreto 6.949/2009 e das normas contidas na NBR 9050/2015 para que as pessoas com deficiência possam ter garantidos seus direitos de acessibilidade sem o impedimento de barreiras arquitetônicas e de comunicação e exigir que tal legislação seja cumprida.

Conclusão

A partir do exposto, podemos constatar que as pessoas com deficiência não dispõem de condições plenas de acessibilidade adequada nas UBS contempladas no presente estudo.

Sendo assim, é necessário que haja uma sensibilização da sociedade civil como um todo, assim como das autoridades, para que desta forma sejam cumpridas as legislações que tratam da acessibilidade na atenção básica e demais espaços públicos e privados, em conformidade com o princípio de universalidade de acesso do SUS.

O fato de o poder público municipal não garantir direito de que pessoas com deficiência tenham acesso pleno aos serviços de saúde, particularmente, os da Atenção Básica, demonstra como ainda vigora preconceito para com este segmento da sociedade. Ficou evidenciada distância oceânica entre as peças legais em vigor no Brasil e a realidade discriminatória predominante em nossas cidades, estampadas na injustificável falta de acessibilidade em $51 \%$ das UBS investigadas. Tanto quanto inadmissível a constatação de que as portas dos banheiros masculinos e femininos impedem entrada de cadeirantes, além de surdos e cegos indisporem de condições básicas de comunicação e orientação espacial.

Mais pesquisas se fazem necessárias, para que novas evidências possam apresentar o panorama da acessibilidade das pessoas com deficiência em outras localidades, além de serem incentivadas a realização de encontros, fóruns e eventos para expor e propor soluções para esta situação, pois o comprometimento do deslocamento das pessoas com deficiência afeta, sobretudo, a qualidade de vida desses indivíduos.

É necessário que sejam implementados mecanismos efetivos e sistemáticos de fiscalização do cumprimento da legislação vigente, para que pessoas com deficiência não sofram discriminação e constrangimentos que, segundo a Convenção das Nações Unidas sobre Direitos da Pessoa com Deficiência, constitui crime, a ser punido na forma da Lei. Ademais, compete aos Conselhos Municipais de Direitos da Pessoa com Deficiência a função deliberativa de fiscalizar o cumprimento da Lei, tanto quanto do acionar o Ministério Público para resolver casos recorrentes.

Este estudo não esgota a análise e discussão da temática acessibilidade para pessoas com deficiência nos serviços de saúde, em particular, aqueles prestados pelas equipes da Estratégia Saúde da Família (ESF), atuantes na Atenção Básica, até porque as UBS, como entrada do sistema de saúde no âmbito do SUS, devem encaminhar esses usuários para atendimentos na Rede de Cuidados da Pessoa com Deficiência. Cabendo aos enfermeiros avaliar as condições de acesso de todos os clientes da população-alvo da UBS em que atuam, garantindo assistência domiciliar às pessoas com deficiência, mais especificamente as concernentes às habilidades para o autocuidado e atividades cotidianas, considerando se tratar de público potencialmente candidato aos cuidados de longo prazo. 
1. Bernardes LCG, Maior I, Spezia CH, Araujo TCCF. Pessoas com deficiência e políticas de saúde no Brasil: reflexões bioéticas. Ciênc Saúde Coletiva 2009;14(1):31-8. https://doi.org/10.1590/s1413-81232009000100008

2. Instituto Brasileiro de Geografia e Estatística (IBGE). Censo Demográfico: Resultados preliminares da amostra [Internet]: 2010. [citado 2018 Fev 9]. Disponível em: http://www.ibge.gov.br/home/estatistica/populacao/censo2010/resultados preliminaresa mostra/default resultados preliminares amostra.shtm

3. Machado WCA. O cotidiano na perspectiva da pessoa com deficiência. Curitiba: CRV; 2017.

4. Machado WCA, Pereira JS, Prado ARA, Silva RA, Silva VM, Figueiredo NMA. Comunicação de profissionais de unidade de reabilitação física com clientes surdos. Rev Rene 2015;16(5):649-55.

5. Associação Brasileira de Normas Técnicas (ABNT). NBR 9050:2015. Acessibilidade a edificações, mobiliário, espaços e equipamentos urbanos. Rio de Janeiro. 2015. Terceira Edição. 162p. Disponível em: http://www.pessoacomdeficiencia.gov.br/app/sites/default/files/arquivos/\%5Bfield gener ico imagens-filefield-description\%5D 164.pdf

6. Brasil, Decreto no 6.949, de 25 de agosto de 2009. Promulga a Convenção Internacional sobre os Direitos das Pessoas com Deficiência e seu Protocolo Facultativo, assinados em Nova York, em 30 de março de 2007. Diário Oficial da União - Seção 1 - 26/8/2009, Página 3. Disponível em: http://www.pessoacomdeficiencia.gov.br/app/sites/default/files/publicacoes/livrolegislacao-federal-sobre-os-dpd.pdf

7. Oliveira LS, Almeida LGN, Oliveira MAS, Gil GB, Oliveira CAB, Medina MG et al. Acessibilidade a atenção básica em um distrito sanitário de Salvador. Ciênc Saúde Coletiva 2012;17(11):3047-56. https://doi.org/10.1590/s1413-81232012001100021

8. Mendes EV. As redes de atenção à saúde. Ciênc Saúde Coletiva 2010;15(5):2297-305. https://doi.org/10.1590/S1413-81232010000500005

9. Brasil. Conselho Nacional de Saúde. Resolução ํㅡ 466/12, de 12 de dezembro de 2012. [citado 2015 Fev 9]. Disponível em: http://conselho.saude.gov.br/resolucoes/2012/Reso466.pdf

10. Castro SS, Lefèvre F, Lefèvre AMC, Cesar CLG. Acessibilidade aos serviços de saúde por pessoas com deficiência. Rev Saúde Pública 2011;45(1):99-105. https://doi.org/10.1590/S0034-89102010005000048

11. Almeida MHM, Pacheco S, Krebs S, Samelli A, Molini-Avejonas DR et al. Primary health care assessment by users with and without disabilities. CoDAS 2017;29(5):e20160225. https://doi.org/10.1590/2317-1782/20172016225

12. Martins KP, Costa TF, Medeiros TM, França ISX, Costa KNFM. Internal structure of Family Health Units: access for people with disabilities. Ciênc Saúde Coletiva 2016;21(10):3153-60. https://doi.org/10.1590/1413-812320152110.20052016

13. Oliveira ALM, Resende MC. Oficinas vivenciais: reflexões sobre direitos humanos de pessoas com deficiências. Psicol Esc Educ 2017;21(2):295-301. https://doi.org/10.1590/2175-3539201702121118

14. França ISX, Pagliuca LMF. Acessibilidade das pessoas com deficiência ao SUS: fragmentos históricos e desafios atuais. Rev Rene 2012;9(2). https://doi.org/10.15253/rev\%20rene.v9i2.5050

15. Bonello AALM, Correa CRS. Acesso aos serviços básicos de saúde e fatores associados: estudo de base populacional. Ciênc Saúde Coletiva 2014;19(11):43974406. https://doi.org/10.1590/1413-812320141911.13922013

16. Santos W. Deficiência como restrição de participação social: desafios para avaliação a partir da Lei Brasileira de Inclusão. Ciênc Saúde Coletiva 2016;21(10):3007-15. https://doi.org/10.1590/1413-812320152110.15262016

17. Cunha ABO, Silva LMV. Acessibilidade aos serviços de saúde em um município do Estado da Bahia, Brasil, em gestão plena do sistema. Cad Saúde Pública 2010;26(4):725-37. https://doi.org/10.1590/S0102-311X2010000400015

18. Amaral FLJS, Holanda CMA, Quirino MAB, Nascimento JPS, Neves RF, et al . Acessibilidade de pessoas com deficiência ou restrição permanente de mobilidade ao 
SUS. Ciênc Saúde Coletiva 2012;17(7):1833-40. https://doi.org/10.1590/S141381232012000700022

19. Pereira JS, Machado WCA. Referência e contrarreferência entre os serviços de reabilitação física da pessoa com deficiência: a (des)articulação na microrregião Centro-Sul Fluminense, Rio de Janeiro, Brasil. Physis 2016;26(3):1033-51.

https://doi.org/10.1590/s0103-73312016000300016

20. Brasil. Ministério da Saúde. Portaria no 339, de 4 de março de 2013. Redefine o Componente Ampliação do Programa de Requalificação de Unidades Básicas de Saúde (UBS) [citado 2016 Nov 1]. Disponível em:

http://www.lex.com.br/legis 24230424 PORTARIA N 339 DE 4 DE MARCO DE 2 013.aspx 